\title{
Data Analysis and Data Sensing Modules for WSN using Verilog
}

\author{
Gudimella Krishna Kishore, GollakotaVenkata Krishna Sharma, Bonthu Brahma Reddy
}

\begin{abstract}
Wireless Sensing Networks (WSNs) are allocated choosing up ecological communities prepared with computational intelligence in addition to radio discussion competencies. The 'nerve cells' of a ordinary WSN as layout module could provide nodes expected for every and each sensing unit which can be miniature, lesser price, effective modeling for different assets with presenting extraordinarily extremely-speedy deployment, versatility and strength-efficient in current style techniques. Wireless Sensing Networks for business objectives or I-WSNs have sure requirements to achieve load for every and every sensors so one can be placed around the plant to test the design managing elements, for troubleshoot and also upgrade the called for test logs. In current layout, we gift a complete custom format of a sensor node for Industries providing Wireless Sensing Networks with the number one emphasis at the constructing elements of the utility. Our style passes at the additives of facts driven changes on components as 6-channel 12-bit Delta-Sigma ADC, a FSM-modular controller thinking about interfaced serial and same M-S (grasp and servant) operated interfaces where trans-receiver is layout with OFDM RF Tx. Present designed estimates an unique modular shape and also improvisator to utilize OFDM based totally upon the RF signaling for OFDM subsystem attending to tracking for a ways better reliability in Wireless Sensing Networks. This made tool has actually long past via Xilinx FPGA Airtex-6 with Front-case RTL layout in addition to modeling to simulate in addition to synthesize using a selection of EDA equipment and the following outcomes have been severely checked in this paper.
\end{abstract} Verilog

Keywords : FPGA , OFDM, Wireless Sensing Networks,

\section{INTRODUCTION}

Prior to region name of Wireless Sensing unit Networks (WSNs) would be short ended up being an alternatively demanded region typically due to the massive choice of researches concluded and applied in real time criteria's to enable the design embedded features. Wireless Sensing devices and its design for Networks would estimate larger and exceptional aspects to provide additionally amazing offers to sensing gadgets (if no greater additional), released throughout the facility of price of hobby in order to show, keep and restore vital operations. The important intricacies of sensing devices in wireless region from the vital fact that Sensor Style similarly to relevant Networking entail layout restraints in methods for communicating different devices in wireless region, Concept's and relational methods for

Revised Manuscript Received on December 13, 2019.

* Correspondence Author

Gudimella Krishna Kishore *, Research Scholar, Dept of ECE, Gitam Deemed to be University, Vishakhapatnam, India.

Email: krishnakishore414@gmail.com

Assistant Professor, Dept of ECE, VignanaBharathi Institute of Technology, Hyderabad, India.

GollakotaVenkata Krishna SHARMA, Professor, Dept of ECE ,Gitam Deemed to be University, Vishakhapatnam, India.

Bonthu Brahma Reddy, Professor, Dept of ECE, VignanaBharathi Institute of Technology, Hyderabad, India. Email: reddybb@ hotmail.com
Email: sharma.gollakota@gitam.edu

networking, Protecting set of rules and list of criteria of design model, and additionally modeling the system and to manufacture the devices, triggering researchers inclusive of students, engineers etc.., to observe several real time parametric criteria associated with their huge-scale implementation. Nevertheless, modern technological era in semiconductor-devices and communicating wirelessly in specific region have brought about notably dependable, durable and finances friendly sensor nodes helping with a miles-desired global outreach allowing common deployment, likewise accelerating capacity packages of WSNs.

Understanding the ones factors of WSNs, the studies furnished on this paper rotates round the format and additionally protection of a sensing unit node for Industrial WSNs even as applying cut as a tradeoffs that are inherent in it. The sensing unit node shape targeted in this text makes up a six level of channeling for design of 12 bits Sigma delta Converter for analog and digital case, a FSM modelling for controlling specific inputs to monitor and test, an interface module based on serial and parallel scenario with master-slave operation for frequency division based on orthogonally multiplexing of trans-receiver module. The current design would provide a simpler model of 4 channeling of conversion in A-D provides a criteria for implementing afour particular sensing gadgets (Temperature sensors, Pressure sensing gadgets, and many others.) In order to deliver an opportunity approach to the tracking expertise's of the node. The sensing unit node format focuses on a long way off surveillance of different scenarios for each plant and its parametric criteria, consider a specific scenario where each nodes accumulated for sensing data may be used to adapt the records concerning the governed temperature-scene and moreover pressure-model for internally and control a generator, moreover report the ones specs to a surveillance station for in addition motions, if desired. [1] The controller subsystem promotes the operation of various statistics switch most of the proposed converter for A-D and subsystem for modeling a Tx-Rx for radio-frequencies trans-reception. The proposed subsystem for modeling Tx-Rx in radio-freq region permits virtual model for processing base-band information actuate from the controller to provide as trans-receiver for the current station in regional aspects. Although an entire considerable amount of researches exist within region and location of Wireless Sensing devices and networking criteria's, there may be a loss of literary works on the surrender-to-surrender layout of an entire sensing unit node for wireless units in current industrial sector and its network regions. Currently, we impart the structure and design model aspects of a Converter for A-D, a controller operated via state-model and its real time scenario with primarily estimating the node access in each scenario where the subsystem would eventually provide the interfaces on frequency modeled transmission optimizable 


\section{Data Analysis and Data Sensing Modules for WSN using Verilog}

(this is deployed within the industrial business enterprise surroundings) and the a ways flung tracking station. These write-up facts a modular structure defined via three essential capabilities that are extraordinarily applicable in these days WSN panorama. Current design scene for the first criteria we arrange a multiplexer in the converter for A_D subsystem promoting a six-channel sub-system of the Delta-Sigma conversion layout in reliability, i.e. A big choice of sensing devices can be attached to the device allowing multiple trackers of boundary-less performance in financial environments entirely. Meanwhile, the subsystem in radio-freq band would endorsed format makes up an processing the base-band for implementing the frequency model for orthogonal multiplexing issue which debts for the dependability and performance of interactions many of the nodes in sensing region and the tracking terminal. Ultimately, to manage the current-subsystem we employ a state model based controller design layout where the parametric criteria such as size, physical length and smaller software program software in addition to facts location.

\section{ARCHITECTURE AND SYSTEMS MODEL}

Wireless Sensing Networks improvise an organization of dispersed self-reliant sensing units in spatially to keep an eye on varied parameters such as temperature level, stress and anxiety, acoustics, rate, photos, and so on. Conservatively, the data-info along to the crucial stations where each data have to be monitored. As per the self-sufficient components labeled 'sensing unit nodes' frequently include the succeeding components:

1. Transceiver in radio-freq region

2. Controller via State modeling

3. Circuit to interface the sensing unit with a sustainable electrical-energy source and to pre-process data info with current scenario.

[2] We impart the criteria on parameters for A-D conversion to collect details from analog sensing units tracking the plant surroundings and transform them right into their equal digital illustration for in a similar way processing). The records gathered are to process and implement by modeling the features of nodes and also sent to the adjacent-node in the area. Currently, the operating-nodes would cooperate in adjacent region and current region bypassing along the records to the output status node which after that transfers the info to exiting necessary tracking station. Apart from the current devices working on the wireless regions and shows a structures in doubling the directions i.e. at one place two directions making for possible to provide far flung get access to as well as handle of sensing unit leisure activity. The dimension and also expense restrictions associated with sensor node design enforces equivalent restrictions on different style functions such as reminiscence, power consumption, computational pace as well as transmission capacity. The capacities of a usual sensing unit node include, Strength, Minimum strength consumption, Wheelchair as well as Heterogeneity, Scalability as well as Affordable. Together with those typical qualities, a sensor node ought to deal with severe environmental problems, need to be easy to install and also hold and also most of all the transmission abilities have to particular protection actions, particularly in the military as well as industrial scenes and places.

[3] Wireless sensing nodes would stand for a sensing unit subsystem, a controller via finite machines as state modeling and trans-receiver for a gadget in different events criteria to operate a different module in one environment such as Tiny-OS. Sensing for different nodes devices in wireless region and network surrounding would be based on plans calls for implementing different real-time aspects both scenario's where hardware module and software modeling are the important task and key aspects. The methods in accordance with $\mathrm{S}-\mathrm{H}$ are in the style where each method can be a design module and application scenario which are unique or generalized. Consider a unit, [4] where each model for VLSI structure for a node to be accessed as sensor are utilized in a image sensing devices in wireless region and its surrounded network aspects. In contrast, the design criteria and its implementing factors for the SOC would be detailed in [4] is composed of a preferred-reason ingrained controller alongside with devoted equipment accelerators for photo handling and also Wi-Fi conversation. In evaluation to this, [6] speak about an adjustable modular structures and its parametric models to promote the circuits in accordance with adders and subtractions considering to handle events and its active and non-active scenarios for devices present in wireless region which are access for sensing data info, therefore imparting an additional generalized topology of sensor-node format. Nonetheless, each highlight on using hardware-primarily based performances to promote quicker and also extra-efficient implementation of computationally extensive responsibilities.

\section{EXISITNG MODEL}

\section{- Modeling for driving the different Data}

[4]WSN programs are all approximately sensing facts in a surroundings and translating this into useful records for the surrender-consumer. So, absolutely all WSN utility are characterized through nearby processing of the sensed information.

- Larger to smaller model for transmissions:

Given that transit on the radio freq would be genuinely steeply-priced in regards to durability, considering the need to storing a lesser so that it will make larger node lifetime. Information communication want to be establish the node to preserve sturdiness, such a number of sensing different values will be inflected to statistics values.

- Designing a specific circuit for single application:

Estimating the circuits which would "one-length-fits-all" be providing all requirements when you consider that a widespread motive CPUs in which additionally power lower and usages for the sensor node's restrained energies on power consumptions in addition to estimations for diverse charge arrays. ASICs, but, are added strength pushed models which affects cut price and absence of the electricity to assist with lots of definitely one in all a kind programs.

- Data Accessing and Addressing inflicting in Memories:

Considering the current list of processors and controllers in different domain of applications where the divide and rule technique would emphasize on each design module for higher speed accuracy for processing different data and control related applications. Yet due to this famous method, algorithms are taken into consideration to spend as a lot as 
40-- $60 \%$ of the instant in having accessibility to memory, making it a site visitors jam. Furthermore, in coinciding the design criteria operations related to specific instructions would efficiently execute required list of the designed parametric criteria.

- Principles for data flow model and control flow model

Managing realities movement (to or from facts reminiscence) and the direction movement (for memory related to set of instructions) as in middle valuable device, methods exist. Controlled drift, the documents float is an end result of the education activity, at the similar time as underneath information waft the education and learning flow is an influence of the documents circulation into. To additional or a lot less choose the order of execution.

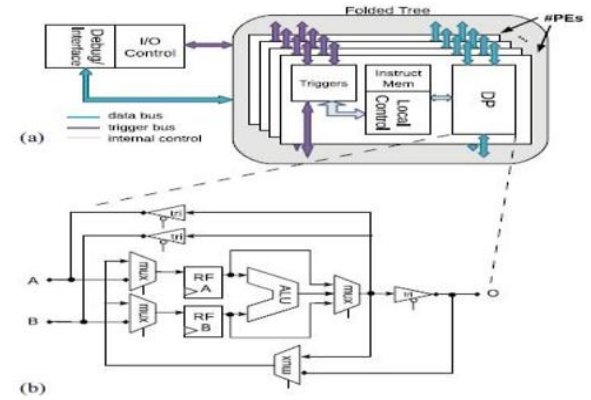

Fig.1. Representing the Control and Design model for $T x$ and $R x$ in WSN.

\section{PROPOSED SCENARIO}

In the present machine, they utilized binary tree for the records dealing with. The binary tree is folded to extend a binary folded up tree for the reuse of dealing with components. In the electronic format globe, prefix procedures are high-quality understood for his or her utility among deliver appearance- earlier adders. The enhancement of two inputs An and additionally B in this situation consists of three degrees. A bitwise propagate-generate (PG) commonplace sense segment, a collection of logic section, and a sum degree. The output of bitwise PG degree is obtainable below.

$P F_{i}=A_{i}$ xor $B_{i}$

$X_{i}=A_{i} * B_{i}$

Group PF-X states for different logical values, modeling the current equation as above:

$P F_{i} X_{i}\left(P F_{i}+1, X_{i}+1\right)=\left\{P F_{i} \cdot P F_{i}+1, X_{i}+P F_{i} \cdot X_{i}+1\right\}$

\section{A.PREFIX ALGORITHM}

Prefix approaches can be computed in a variety of methods, though we picked the binary tree method as a result of the truth its slide suits the desired on-the-node records aggregation. This may be imagined as a binary tree of dealing with components (PEs) at some stage in which enter facts moves from the leaves to the foundation. This topology will shape the set part of our approach, though for you to provide greater than one programs, versatility is likewise required. To lessen the energy consumption similarly, the PE's ought to make use of better superior.

Via a few constructing adjustments has truly been accomplished on Tx and Rx Design for WSN, the energy may be decreased likewise. The tree-based data flow will, finally, be finished on a stats direction of programmable PEs, which provides this versatility at the same time with the parallel prefix principle. In prefix set of tips, 2 stages are utilized.

- Trunk phase

- Twig phase

\section{Trunk phase}

In the trunk section the left charge $\mathrm{L}$ is stored regionally as $\mathrm{L}$ save as properly as it's lots introduced to the appropriate charge $\mathrm{R}$, this is exceeded on inside the route of the idea as confirmed in fig 2 (a). The operation of the structure would be reprinted with its design of the adder or multiplier structure for the transform block in Sensor networking of wireless devices. Each scenario of the addition would estimate the different structural changes for the prescribed operations.

\section{Twig phase}

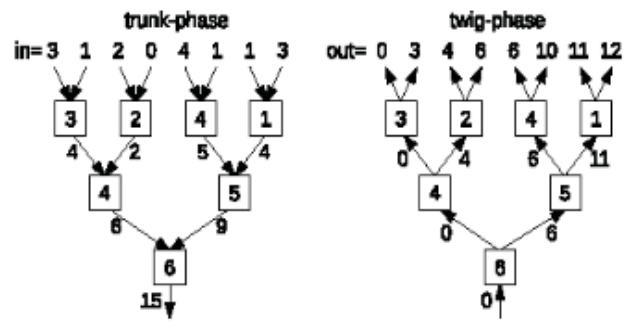

Fig. 2. (a) (b) Model for trunk and twig phase

The branch area starts, inside the instructions of which realities movements within the contrary path, from the basis to the leaves as confirmed in fig 2 (b). Now the incoming rate, starting with the amount identification aspect 0 at the muse, is exceeded to the left child, at the same time as it is additionally presented to the previously conserved Lsave and handed to the appropriate baby. In the cease, the reduced prefix collection is discovered at the leaves.

\section{FOLDED TREE}

The idea provided right here is to provide a scenario for operating the reverse tree-structure and its folded operation would recycle the PEs. Subsequently, the functionality of the processed elements in the current design stated for controlling and transforming the data would be directly related to half of the no of the bits utilized on design criteria. On behalf the additional model for controlling and processing tree scene is lowered. The current model for utilizing the folded approach is displayed in fig 3 .

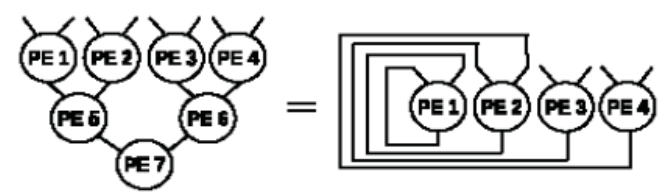

Fig.3.Repressing the binary tree for folded approach The processing elements consists of the following:

$\square$ FSM-Controller

Instruction memory

Triggers

Local control 


\section{Data Analysis and Data Sensing Modules for WSN using Verilog}

Taking into consideration the existing design for common method in which each such prefix motive force for approximate exact same additions has actually been configured to do on the folded up tree. As an instance, the Summation goes to enforce a prefix amount for equivalent system on the processed elements where each element is hatched by adjoining of the other elements in the considered tree. The organized comparable binary tree in the middle throughout once again suggests exactly just how details tasks from fallen leaves to root all through the trunk-section. The tree folded approach would estimate its structures and represents the corresponding elements as " $L$ "and " $R$ " as the left and right state events observed from the top node of the tree where the inputs An and Bn would be the elements considered at each section of the "L" and "R".For such changes observed in the tree model we expect the output changes in each section of the T-child and its derivatives accordingly. Each such derivatives are processed and stored in time $\mathrm{Tx}+\mathrm{Rx}$.

The first degree has all 4 PEs vibrant. The 2nd phase has 2 energized PEs: PE3 as well as also PE4. The third as well as last diploma has simplest one dynamic PE: PE4. A lot more notably, it might moreover show up that PE3 in addition to PE4 need to plainly keep more than one Lsave values. PE4 demand to protect 3: Lsave0 thru Lsave2, also as PE3 maintains 2: Lsave0 and Lsave1. PE1 as well as PE2 every merely preserve one: L_save0. Considering the results for the expected trunk level phase at each folded approach prefix additions for each adder would estimate an prefix-sum approach for trunk stage and folded stage.

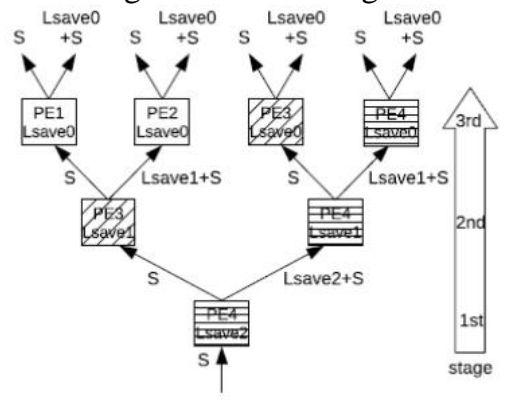

Fig.4.Repressing the stages for twig model

The trunk-segment PE software program modeling listed below has three commands, which may equal, in addition to the exceptional RF addresses which might be made use of. Now, the twig-section is thought about the use of Fig. 4. The tree runs inside the contrary commands, so an inbound charge (repressed to assign as S) gets in processed Element via a port for the output scenario. Adhering to proposed technique, $S$ is gathered and obligated to assimilate in all directional for the time in $(S+$ Lsave)is subjected to move in right direction. Hence, the elements which need to be processed are became on during the twig-segment once more affects precisely exactly how the programs of the folded tree demand to take place. To explain this, each section of the twig-segment are revealed collectively to higher see simply exactly how every $\mathrm{PE}$ is activated throughout the twig-segment as well as for what variety of degrees. Initially, an incoming price (in this case the acknowledgment detail S2) is exceeded to the left. Afterwards it's miles offered the formerly (from the trunk-segment) preserved Lsave2 charge and likewise went beyond to the correct. PE4-training 1 will genuinely both pass the amount Lsave $2+\mathrm{S} 2=\mathrm{S} 1$ to the right (= itself) and miss this $\mathrm{S} 1$ furthermore the left within the instructions of PE3.

\section{RESULTS AND DISCUSSIONS}

This paper supplied the folded tree design of an virtual model for the design model for the main subsystems such as memory and controller programs. The style exploits the fact that numerous designs would tend to provide the structural and algorithmic aspects of the current model in wireless devices for sensing applications can be defined making use of parallel-prefix operations, supplying the plenty wished adaptability. Power consideration for storage structured as:

1) Given data information and its implementation with prefix approach for parallel operations and its processing.

2) Folded approach for each nodes representation and data accessing

3) The blend of records glide in addition to control flow additives to give a community dispersed memory, which removes the reminiscence web site visitor's jam even as maintaining enough flexibility. The simulation outcomes are shown in under.

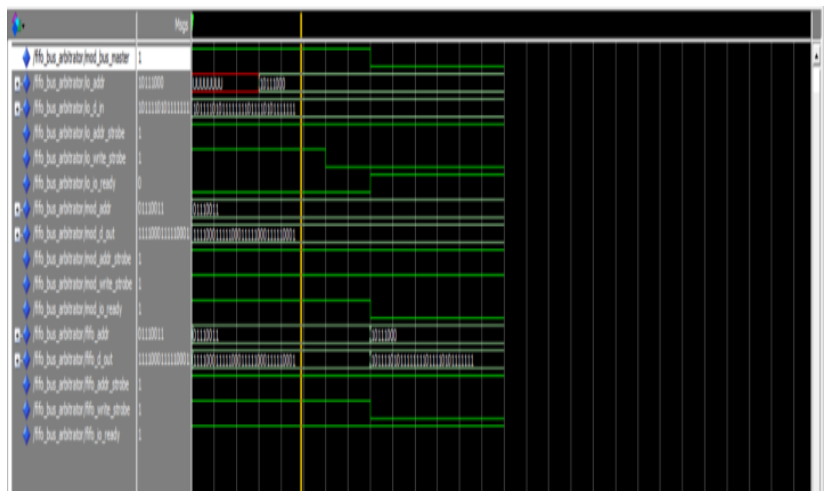

Fig.5.FIFO Control And Memory For WSN.

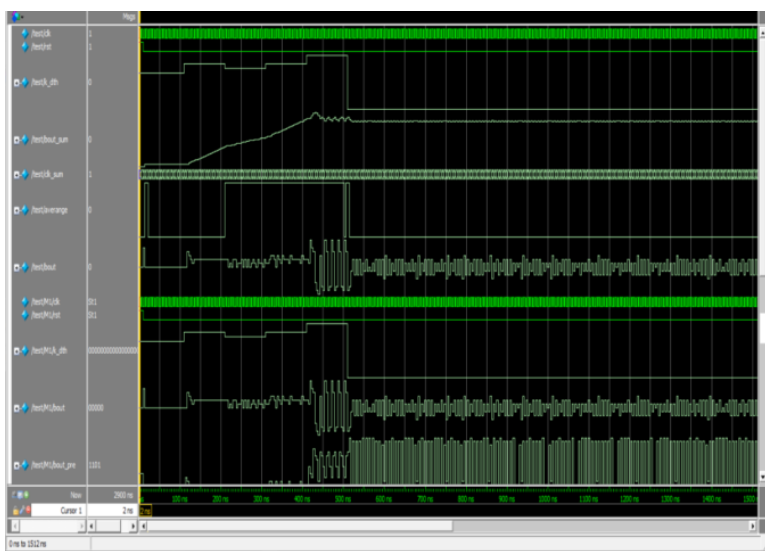

Fig.6. Representing an ADC \{Prefix Modeling\} For WSN Tx And Rx.

\section{FUTURE ENHANCEMENT}

The future Extent of this job goes to the quit of format router is consisted of. It is used to reduce the maintain-up together with blockage. Also the novel designs may be decided for the complex facts managing

\section{ACKNOWLEDGMENT}

The following work has been carried out using computational facility provided by DST's FIST lab in VBIT, Hyderabad. India. 


\section{REFERENCES}

1. C.Walravens and W. Dehaene, "Design of a low-energy data processing architecture for wsn nodes," in Proc. Design, Automat. Test Eur. Conf.Exhibit., Mar. 2012, pp. 570-573.

2. H. Karl and A. Willig, "Protocols and Architectures for Wireless Sensor Networks”, 1st ed. New York: Wiley, 2005.

3. S. Mysore, B. Agrawal, F. T. Chong, and T. Sherwood, "Exploring the processor and ISA design for wireless sensor network applications," in Proc. 21th Int. Conf. Very-Large-Scale Integr. (VLSI) Design, 2008, pp. 59-64.

4. P. Sanders and J. Träff, "Parallel prefix (scan) algorithms for MPI," inProc. Recent Adv. Parallel Virtual Mach. Message Pass. Interf., 2006, pp. 49-57.

5. G.Blelloch, "Scans as primitive parallel operations," IEEE Trans.Comput., vol. 38, no. 11, pp. 1526-1538, Nov. 1989.

6. N. Westeand D. Harris, "CMOS VLSI Design: A Circuits and Systems Perspective. Reading”, MA, USA, Addison Wesley, 2010.

7. M. Hempstead, D. Brooks, and G. Wei, "An accelerator-based wireless sensor network processor in $130 \mathrm{~nm}$ cmos," J. Emerg. Select. Topics Circuits Syst., vol. 1, no. 2, pp. 193-202, 2011.

8. B.A Warneke and K. S. J. Pister, "An ultra-low energy microcontroller for smart dust wireless sensor networks," in Proc.IEEEInt. Solid-State Circuits Conf. Dig. Tech. Papers. Feb. 2004pp. 316-317.

9. M. Hempstead, M. Welsh, and D. Brooks, "Tinybench: The case for a standardized benchmark suite for TinyOS based wireless sensor network devices ," in Proc. IEEE 29th Local Comput. Netw. Conf., Nov. 2004, pp. 585-586.

\section{AUTHORS PROFILE}

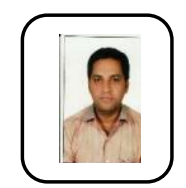

Gudimella Krishna Kishore has completed B.Tech and M. Tech in the year 2009 and 2011. Now Pursuing Ph.D. in Wireless Sensor Networks and VLSI Design from Gitam Deemed to be University under the superviosn of Dr. G.V.K. Sharma, Professor ,Department of ECE.

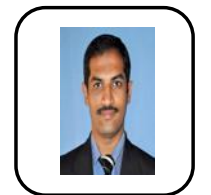

G.V.K. SHARMA obtained his Ph.D. and Master's degree from Andhra University and Indian Institute of Science, Bangalore respectively. Presently, he is a Professor in the Dept. of ECE, GITAMDeemed to be University, Visakhapatnam, India. His research interests include radar signal processing, VLSI signal processing and multicarrier communications.

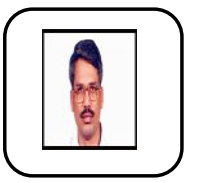

Bonthu Brahma Reddy has completed his M. Tech from IIT-Madras in the year 1982 and Ph.D. in the year 2016 from JNTUH. His research domain includes Wireless Sensors/Ad-hoc Networks and Wireless Communication. 